\title{
Conductive Polymers for Ammonia Sensing: Electrodeposition, Hybrid Materials and Heterojunctions
}

\author{
M. Bouvet $^{1}$, M. Mateos ${ }^{1}$, R. Meunier-Prest ${ }^{1}$, J.-M. Suisse ${ }^{1}$ \\ ${ }^{1}$ Institut de Chimie Moléculaire de l'Université de Bourgogne, UMR CNRS 6302, \\ Université de. Bourgogne Franche-Comté, Dijon, France \\ marcel.bouvet@u-bourgogne.fr
}

\begin{abstract}
:
Polyaniline (PANI) with electron-donating and electron-withdrawing substituents were electrodeposited and studied as sensing materials in conductometric transducers. Whereas the dimethoxyaniline leads to a highly conductive material, the tetrafluoroaniline leads to a poor conductive polymer. However, it was used in heterojunctions. Elsewhere, hybrid materials combining polypyrrole (PPy) with ionic macrocycles as counterions were also electrosynthesized and used as sensing material for the detection of ammonia. They exhibit a higher sensitivity compared to PPy prepared with small counterions, with a stable response in a broad range of relative humidity and at room temperature.
\end{abstract}

Key words: conductive polymers, conductometric transducer, heterojunction, ammonia, relative humidity.

\section{Motivation and results}

A strong demand exists for gas sensors operating at low temperature and prepared with low energy processes. Thus, molecular materials, including polymeric materials that can be often deposited by solution processing, are good candidates. They can also be used to prepare hybrid materials when associated with carbonaceous or inorganic compounds. The properties of the obtained materials, e.g. morphology, roughness and specific surface, hydrophilicity or hydrophobicity and eventually their optical and electronic properties can thus be tuned to optimized their sensing properties. Among solution processing technologies, electrodeposition presents net advantages, for example when different materials need to be deposited on different electrodes located on the same substrate.

The electronic properties of polyaniline derivatives [1] are highly dependent on the nature of the substituents on the aniline ring. Numerous studies have focused on the properties of monofluorinated derivatives [2], but only a few on the fully fluorinated polyaniline (PolyTetraFluoroAniline PTFA). The object of our work was to carry out a complete study of the PTFA polymer. First, a UV-visible monitoring of the growth of polymer films by chrono-amperometry was performed on a gold grid. Then a electrochemical study of the monomer (Fig 1) and the polymer was carried out on glassy carbon (GC). PTFA was also deposited on ITO substrate and characterized by SEM (Fig. 2) and XPS, and compared to PANI. Due to the fluorine atoms that limit its oxidation, PTFA is a poor conductive material compared to PANI.

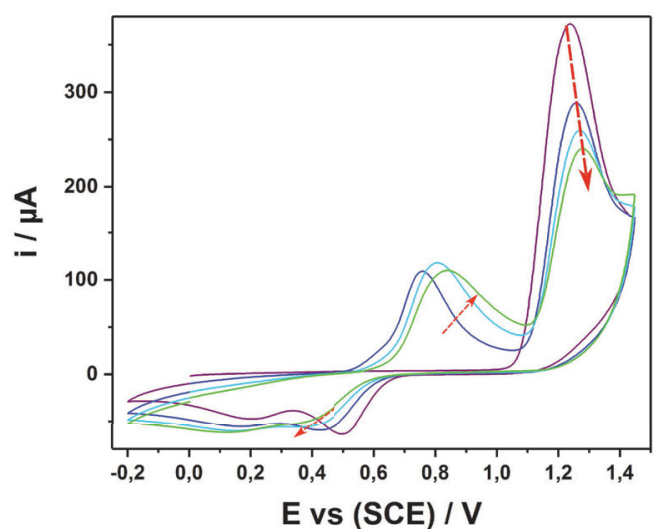

Fig. 1: Cyclic voltammetry on glassy Carbon of TFA $50 \mathrm{mM}$ in $\mathrm{HClO}_{4} 2 \mathrm{M}, \mathrm{V}=0.1 \mathrm{~V} \cdot \mathrm{s}^{-1}$, allowing the electrodeposition of a PTFA film.

However, we took advantage of this unique behavior to build heterojunctions, by 
combination with a highly conductive molecular material, namely the lutetium bisphthalocyanine. The obtained double lateral heterojunction exhibits particularly interesting sensitivity to ammonia, even in humid atmospheres [3], with a behavior near this of molecular semiconductor - doped insulator heterojunction previously reported [4].
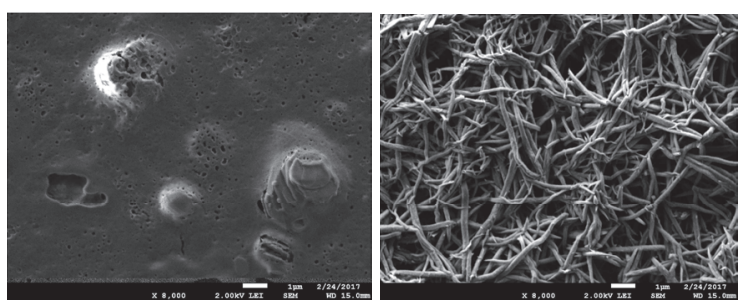

Fig. 2: SEM images of PTFA (left) and PANI (right) electrodeposited on ITO.

We also electrosynthesized a PPy material with a ionic phthalocyanine as counteranion with a particular morphology (Fig. 3) and a stable response in a broad range of relative humidity (Fig.4) [5].

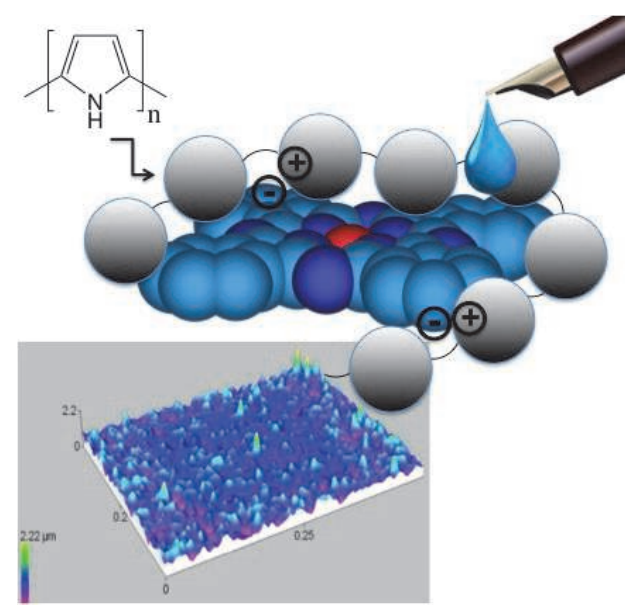

Fig. 3: Schematic view of the ionic interactions between polypyrrole and cobalt sulfonated phthalocyanine (CoTsPc), and a topomicroscopic view of the hybrid material.

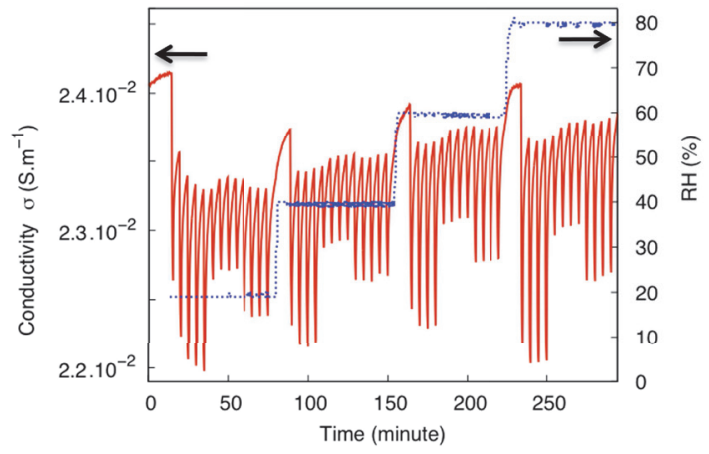

Fig. 4: Conductivity variation of a PPy/CoTsPc resistor as a function of time when exposed to $\mathrm{NH}_{3}$ at various rh values, during $1 \mathrm{~min} / 4 \mathrm{~min}$ exposure/recovery cycles [5].
PPy / phthalocyanine - based resistors were also prepared by the layer by layer technique, with a high sensitivity to $\mathrm{NH}_{3}$ quasi stable in a broad relative humidity range (10-70 \%) (Fig. 5) [6].

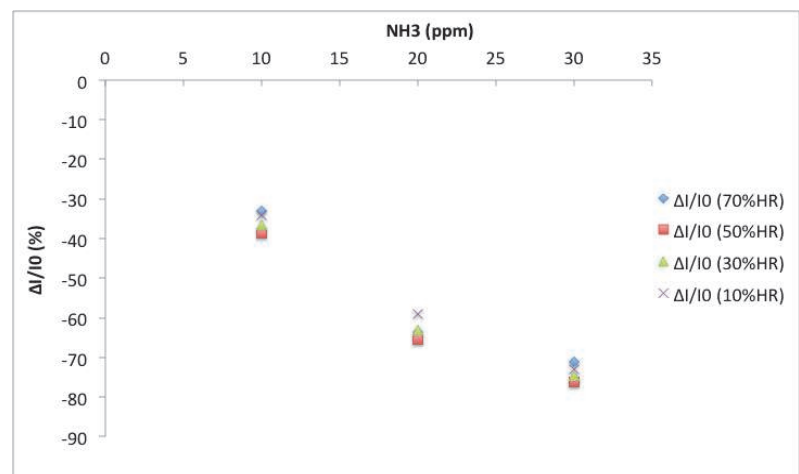

Fig. 5: Relative response $\left(\Delta I / I_{0}\right)$ of a (PANI/CuTsPC) device as a function of the $\mathrm{NH}_{3}$ concentration at various rh values, as calculated from four $0.25 \mathrm{~min} /$ 1 min exposure/recovery cycles.

\section{References}

[1] A. G. MacDiarmid, "Synthetic Metals": A Novel Role for Organic Polymers (Nobel Lecture), Angew. Chem. Int. Ed. 40, 2581-2590 (2001); doi: 10.1002/1521-3773(20010716)40: 14\&It;2581::AID-ANIE2581\&gt;3.0.CO;2-2

[2] L. Astratine, E. Magner, J. Cassidy, A. Betts, Characterization and Electrochromic Properties of Poly(2,3,5,6-tetrafluoroaniline), Electrochimica Acta 74 117-122 (2012); doi: 10.1016/j.electacta.2012.04.032

[3] M. Mateos, R. Meunier-Prest, O. Heintz, F. Herbst, J.-M. Suisse, M. Bouvet, A Comprehensive Study of poly $(2,3,5,6$ tetrafluoroaniline): From Electrosynthesis to Heterojunctions and Chemosensing, submitted.

[4] M. Bouvet, P. Gaudillat, A. Kumar, T. Sauerwald M. Schüler, A. Schütze, J.-M. Suisse, Revisiting the Electronic Properties of Molecular Semiconductor - Doped Insulator (MSDI) Heterojunctions Through Impedance and Chemosensing Studies, Org. Electron. 26, 345354 (2015); doi.org/10.1016/j.orgel.2015.07.052

[5] T. Sizun, T. Patois, M. Bouvet, B. Lakard, Microstructured Electrodeposited PolypyrrolePhthalocyanine Hybrid Material, from Morphology to Ammonia Sensing. J. Mater. Chem. 22, 2524625253 (2012); doi: 10.1039/C2JM35356C.

[6] P. Gaudillat, F. Jurin, B. Lakard, C. Buron, J.-M. Suisse, M. Bouvet, From the Solution Processing of Hydrophilic Molecules to Polymer-Phthalocyanine Hybrid Materials for Ammonia Sensing in High Humidity Atmosphere; Sensors, 14(8), 13476-13495 (2014). doi:10.3390/s140813476 